Recordar cosas del pasado no es necesariamente recordarlas como fueron - Marcel Proust

\title{
Destellos de nostalgia: inmigrantes ideológicos de América Latina en el Israel de hoy $^{1}$
}

Flashes of nostalgia: ideological immigrants from Latin America In today's Israel ${ }^{2}$

\author{
Batia Siebzehner \\ Universidad Hebrea de Jerusalem \\ mbatsib@pluto.mscc.huji.ac.il
}

\section{Resumen}

La práctica de la ideología sionista socialista que caracterizaba a los movimientos juveniles en América Latina implicó para sus miembros emigrar a Israel y vivir en el kibutz. Este artículo analiza la manera en que los grupos de Hashomer Hatzair que emigraron en los decenios de los 50 a los 80 del siglo XX evalúan en la madurez (la segunda década del siglo XXI) la realización de esos objetivos. El cuestionario al que respondieron más de 700 personas que participaron en el movimiento y emigraron a Israel y las entrevistas realizadas a parte de ellos tomaron en consideración los cambios acaecidos en la sociedad israelí desde entonces. Los hallazgos muestran que hay casi un consenso entre los encuestados en lo que se refiere al logro de los objetivos sionistas y a la contribución del movimiento a la conformación de la nacionalidad israelí. Las respuestas que se refieren al kibutz presentan un cuadro más complejo dado que hay quienes ven en esa empresa un fracaso y hay también quienes consideran un posible despertar del socialismo y el colectivo kibutziano.

\footnotetext{
${ }^{1}$ Este artículo se basa en la investigación llevada a cabo por Pablo P. Adi y B. Siebzehner (2008).

${ }^{2}$ Artículo publicado en Cuadernos Judaicos-ISSN: 0718-874 Nº 33 Diciembre 2016
} 
La decepción, e incluso los perjuicios personales parecen no destruir la nostalgia restaurativa con respecto al movimiento juvenil al que le atribuyen un lugar fundamental como vivencia juvenil. Tanto los miembros que aún viven en el kibutz (la mitad de los respondientes) como los que lo abandonaron volverían, con la experiencia actual, a ingresar al movimiento que parece

Palabras claves: Kibutz - Hashomer Hatzair - ideología sionista - sionismo socialista.

\begin{abstract}
The practice of the Zionist socialist ideology that characterized the youth movements in Latín America meant for its members to emigrate to Israel and live in the kibbutz. This article analyzes how the Hashomer Hatzair groups, that emigrated in the 50s to 80s of the 20th century, evalúate the achievement of these objectives in maturity (the second decade of the 21 st century). The questionnaire was answered by more than 700 people who participated in the movement and emigrated to Israel, and the interviews conducted with them took into consideration the changes that have taken place in Israeli society ever since.

The fmdings show that there is almost a consensus among respondents as to the achievement of Zionist objectives and the movement's contribution to shaping Israelí nationality. The answers that refer to the kibbutz present a more complex picture since there are those who see in that company a failure and there are also those who consider a possible awakening of socialism and the kibbutz collective. Disappointment, and even personal injury, do not seem to destroy the restorative nostalgia ith respect to the youth movement, which is credited with a fundamental place as a juvenile experience. Both the members who still live in the kibbutz (half of the respondents) and those who left the kibutz would, with current experience, join the movement that seems to be perpetuating itself as an "Immaculate Disappointment."
\end{abstract}

Keywords: Kibbutz - Hashomer Hatzair - Zionist ideology - Socialist Zionism

\title{
Introducción
}


A finales del año 2016 está prevista la aparición del libro "El camino de un sueño" dedicado a la historia del movimiento Hashomer Hatzair en Argentina. Material similar, aunque diferente en contenido, ya dio luz en referencia al mismo movimiento en Chile (Stoliar, 2003) y en Cuba (Stoliar, 2013). Las narrativas presentadas en esos textos tienen su inspiración en un fenómeno único: la movilización de jóvenes latinoamericanos judíos que emigraron de América Latina a Israel en pro de una ideología que exigió un cambio total de sus formas de vida. Este tipo de inmigración, a la que precedió una formación educativa de varios años, se diferencia de otros casos donde la motivación está mayormente dada por condiciones económicas o políticas en el país de origen. Esta inmigración, en cambio, fue motivada por un acto de consenso ideológico orientado a la creación de la nación-estado judía y a la adopción de una forma de vida comunitaria regida por principios socialistas (kibutz). El intento de tratar de perpetuar a través de la palabra escrita aquellas épocas en las que se forjaron los ideales que dieron forma a la vida adulta parece ser motivado por muchas razones. Para algunos es el deseo de "volver a vivir" aquellas experiencias que dejaron marcadas vivencias juveniles irremplazables otorgando así a la nostalgia un lugar legítimo en la edad mayor. Para otros es, quizás, el deseo de observar a través de la perspectiva del tiempo que significó aquel paso tan dramático. Hay quienes ven en ese acto el cerrar de un círculo que se inició hace más de medio siglo y que, en las palabras de uno de los participantes en las reuniones donde se promovió la edición del libro, dijo que esto les permite "reafirmar el ser en el aquí y en el ahora". Manifestaciones similares se pueden encontrar en el blog donde se anuncia la promoción del libro.

Este artículo trata de analizar la participación en el movimiento juvenil desde una perspectiva diferente que no necesariamente rescata las vivencias del pasado, sino que trata de vislumbrar cómo estas vivencias median en la evaluación de los ideales propuestos por la organización juvenil en la adolescencia. Una de las bases que estimulan al análisis de este fenómeno es el hecho que la ideología sionista socialista, orientada a la creación de un nuevo orden social, atraviesa profundos cambios que en muchos aspectos contradicen las premisas básicas que los inmigrantes ideológicos aspiraban realizar. Israel es un país caracterizado por conflictos externos e internos que ponen de manifiesto un bajo consenso 
sobre componentes de la identidad colectiva (Ben Rafael, 1996). Ideas tales como igualdad, solidaridad, secularismo, socialismo, paz con los pueblos vecinos, fueron reemplazadas por prácticas que ponen de manifiesto tendencias tales como privatización, individualismo, religiosidad, capitalismo, neo-liberalismo, y otras ideas opuestas a los conceptos originales que los ex miembros absorbieron en las filas del movimiento juvenil. Más aún, la misma ideología sionista es puesta en tela de juicio por algunos grupos de la sociedad israelí que consideran que la función histórica ya fue cumplida y ahora hay que redefinir nuevos términos de identidad.

En otras palabras, el tema central que desarrolla este artículo se refiere a la medida en que los ideales de la adolescencia y la juventud, transformados en prácticas de vida, son evaluados en el presente por los protagonistas de lo que algunos de ellos denominaron "epopeya histórica". ${ }^{3}$

El estudio confronta a los individuos con preguntas relacionadas a los ejes centrales que caracterizaron el universo de pertenencia en la juventud y la interpretación de sus consecuencias, tanto a nivel personal como colectivo, en la edad mayor. Esta relación mutua entre la interpretación y las fronteras de acción determinadas por la realidad social parecieran establecer los parámetros básicos capaces de explicar el modo de correspondencia entre la visión retrospectiva de una realidad utópica y la evaluación de los logros personales y colectivos bajo condiciones radicalmente cambiantes (Giddens, 1991).

\section{El movimiento juvenil en el proceso de formación de la identidad}

El impacto de las organizaciones juveniles en la transformación de la forma de vida de los adolescentes es un tema que fue analizado tanto en el mundo judío como fuera del mismo. (Kahane, 2007). Más aún, la historia de la formación del Estado de Israel está íntimamente

3 Tal como definió un ex miembro del movimiento refiriéndose a la transformación de vida resultado de la inmigración a Israel y la adherencia a un kibutz. 
ligada al desarrollo de movimientos que inculcaron en la juventud de la diáspora la idea de la creación de un Estado Judío. Quienes investigaron el desarrollo de los movimientos juveniles judíos en Europa los evaluaron como portadores de un mensaje revolucionario destinado a cambiar a la sociedad judía en general (Alón, 1986; Golán, 1998; Lam, 1991)

La crisis ideológica que se fue gestando en el mundo judío de la diáspora influyó en la consolidación de una cultura juvenil innovadora en cuanto a la forma y el contenido de la misma. Estos marcos jugaron un papel central en la transmisión de las ideas de la modernidad a las comunidades judías y en la traducción de esas ideas a prácticas de vida tanto en la diáspora como en la sociedad israelí (Mintz, 1995; Shapira y Peleg, 1979; Shimoni, 1995).

La investigación académica también pone de relieve el hecho que los movimientos creados en Europa lograron transmitir tanto el mensaje ideológico como la estructura institucional a la juventud que se iba formando en la nueva nación. El análisis pone de relieve la complejidad de la relación entre los marcos que se iban creando en el nuevo medio con la dirección del movimiento en Europa Oriental (Tzur, 2006). Estos agentes de socialización permitieron no solo trasmitir la idea revolucionaria de nuevos contenidos a la identidad judía sino también constituirse en canales de movilidad social gracias a las relaciones con las fuentes de poder político que se iban estableciendo y creando en el Yishuv. (Chen, 1969; Rapaport y Limski, 1995). Los movimientos se constituyeron así, antes y después de la creación del Estado de Israel, en entes de continuidad de los valores y las normas que se fueron institucionalizando en las esferas políticas y sociales. Los movimientos juveniles jugaron además un papel central en el desarrollo institucional de la nación-estado aportando a la movilización de sus miembros a distintas esferas de acción social (Adler, Shapira y Fayer, 1999). Así, el ser miembro de un movimiento juvenil otorgaba un status social muy respetado en la sociedad israelí (Ben David, 1954). 
Los ex miembros de los movimientos les atribuyen a estos una influencia central en las normas, valores y actitudes hacia cuestiones sociales que guiaron sus vidas a lo largo de los años (Verner, 1996). Estas investigaciones, sin embargo, no se relacionaron a la manera en que sus ex miembros evaluaban en la edad mayor las exigencias propuestas por los movimientos en cuanto a la realización de los objetivos. En general mayormente se enfocó el análisis en los movimientos como entidades institucionales más que en el agente que participaba en esos marcos. Más allá de la especificidad de los movimientos juveniles, no hay investigaciones que trataran de analizar el desarrollo del individuo a través del tiempo en relación directa con los ideales que totalizó en su adolescencia. De aquí la importancia de la presente investigación que intenta echar luz al proceso de transformación y/o continuidad de las ideas a nivel individual más que organizacional. Al ubicar al sujeto como actor se trata de entender también la medida en que la transformación de la realidad impacta en la interpretación de la vivencia juvenil. En las palabras de uno de los participantes en las reuniones que intentaban promocionar el libro antes mencionado: "A veces pienso, cómo serían nuestras vidas, sin el encuentro, a veces casual, con el Hashomer Hatzair. ¿Habré Ganado? ¿Habré perdido? ¿A quién le importa? Solo estoy seguro de una cosa: Si me dieran a elegir, viviría nuevamente todos esos años mágicos que viví en el movimiento."

Esta investigación va más allá de entender alimentar la nostalgia juvenil, quiere más bien entender cómo se interpretan a largo plazo las implicaciones de esos "años mágicos" que determinaron el rumbo de vida de los participantes.

\section{La transformación de la Ideología a prácticas de vida}

El participar en un movimiento juvenil en América Latina implicaba un compromiso mucho mayor que el que significaba el participar en los movimientos juveniles en Israel. Allí, los jóvenes que de una manera u otra eran miembros de las organizaciones sionistas pioneras (Jalutzianas) internalizan a través de los años el mensaje que implicaba a largo plazo romper con la vida del país de origen y con los lazos familiares inmediatos para ser 
un agente activo en el proceso de creación del Estado judío. Desde la declaración de Independencia del Estado de Israel en 1948, y aun anteriormente, las comunidades judías en América Latina constituyeron una constante fuente de inmigración. Aproximadamente $3 \%$ de los inmigrantes que llegaron a Israel entre los años 1948-2005 son originarios de esas comunidades ${ }^{4}$, lo que se manifiesta en un número aproximado de 100.000 personas, y se calcula que en la primera década del siglo XXI viven, entre los 5.800.000 de habitantes judíos de Israel, aproximadamente 60.000 personas nacidas en países de América Latina. ${ }^{5}$

El contingente mayor de este grupo, un 64\% del total, procede de Argentina donde residió y reside el mayor número de judíos en América Latina. Hasta los años 70 un sesenta por ciento de los inmigrantes eran jóvenes de entre los 18 y 25 años (Klor, 2016). Dentro de este grupo se calcula que aproximadamente un $18 \%$ eran miembros de movimientos juveniles que llegaron también con el propósito de vivir en los kibutzim ${ }^{6}$, (Bar Guil, 2005). El fenómeno de la inmigración a través de los movimientos juveniles recibió expresión literaria en libros tales como "Una joven política" (2002) donde, desde una perspectiva histórica biográfica, Schechner cuenta sus vivencias en el movimiento Hashomer Hatzair en sus años de adolescencia y en los conflictos ideológicos que vivenció en el kibutz. Rubin (2006), a través de cuentos cortos intenta en su libro hacer partícipe al lector de las experiencias únicas por las que atravesaron quienes participaban en el movimiento. En "Sobre Nosotros" Tromper y otros (2007) recopilaron relatos de miembros del movimiento Ijud Habonim en la juventud. Las investigaciones de Bar Gil (2007) revelan aspectos institucionales de los movimientos Dror, Gordonia e Ijud Habonim al igual que ciertas características del proceso de integración en los kibutzim hasta los años 70. El individuo evaluando su propio camino de vida no fue sujeto de investigación sistemática.

4 Las olas más grandes tuvieron lugar entre 1968-88 (5,1\% de inmigrantes de Argentina y Chile, y 2,5\% de Brasil, de un total de 534.000 inmigrantes); $6,5 \%$ y $1,9 \%$ entre 2001 y 2005 , de un total de $142.489 ; 1,1 \%$ y 0,4\% entre 1999 y 2000 de un total de 1.040.352 (Israel Central Bureau of Statistics, 2007).

5 No hay un número exacto de aquellos que regresaron a su país de origen, que emigraron a otros países o que fallecieron.

6 Comunas que establecen un orden social igualitario en las que se espera que los miembros aportan según sus posibilidades y habilidades y reciban de acuerdo a sus necesidades 
El presente estudio puede considerarse un" análisis de caso": la población investigada proviene del movimiento Hashomer Hatzair calificado como el más "extremista" y radical de los movimientos juveniles de entonces. El movimiento se autodefinía como Sionistamaximalista ya que el emigrar a Israel era una exigencia impostergable a la edad aproximada de 20 años y Socialista-revolucionario ya que no se consideraba ninguna alternativa de vida en Israel fuera del kibutz donde, de acuerdo al discurso predominante, se podía realizar el imperativo de "proletarización". ${ }^{7}$ De este movimiento se estima que llegaron entre 1946-70 alrededor de 1500 olim (inmigrantes), que ingresaron a diferentes kibutzim en base a las decisiones del organismo centralizador del Kibutz Artzi, a cargo de distribuir a los grupos de los inmigrantes de acuerdo a las necesidades de los diferentes kibutzim (Shajner, 2002).

Los datos de la investigación se recolectaron a través de tres métodos, por un lado, un cuestionario de 77 preguntas cerradas diseñadas analíticamente según el Método de las Facetas (Gutman, 1980; Levy 1994) ${ }^{8}$. El cuestionario fue enviado a 712 personas que emigraron a Israel entre 1950-1970 y a quienes se logró localizar por medio de redes sociales. Los cuestionarios fueron devueltos por correo conservando el anonimato. De los 362 cuestionarios que se recibieron de vuelta se confeccionaron dos muestras representativas, una de miembros del kibutz $(\mathrm{N}=187)$ y otra de quienes viven en Israel, fuera del kibutz $(\mathrm{N}=178)$ lo que da lugar a la comparación entre ambas poblaciones, respecto de diversos temas ${ }^{9}$. Otra fuente de datos son las entrevistas que se hicieron con 30

7 No obstante considerarlo case study, no se supone atinada la extrapolación a otros movimientos, a no ser que se realicen investigaciones similares con las poblaciones a comparar. Hashomer Hatzair era considerado por gran parte de la colectividad judía como una agrupación comunista, una percepción que despertaba la oposición de padres a la participación de los hijos en las filas del movimiento.

$8 \mathrm{La}$ característica principal del análisis por facetas, es que éste no trata separadamente observaciones e intercorrelaciones, sino que examina la vinculación (partnership) entre un sistema definidor (definitional system) de un conjunto de observaciones con la estructura de una matriz (multivariable) de las intercorrelaciones.

9 Considerando la complejidad del cuestionario y el hecho de que no todas las direcciones eran correctas, el número de respuestas que se consiguió es de alto alcance en un tipo de investigación de este estilo. Las muestras no son proporcionales al número de personas viviendo dentro y fuera del kibutz pero es de suponer que tanto las direcciones correctas como 1 influencia grupal influyeron en que una proporción mayor de aquellos estuviesen dispuestos a responder. 
ex miembros del movimiento juvenil. Las entrevistas se llevaron a cabo en las casas de los entrevistados y duraron aproximadamente hora y media. El análisis narrativo enriqueció la interpretación de los datos obtenidos a través de los cuestionarios ${ }^{10}$. La tercera fuente fueron observaciones que se obtuvieron a través de la participación en las reuniones organizadas por los generadores del proyecto del libro, con el objeto de promocionar y difundir el proceso de su publicación. Las observaciones contribuyeron a evidenciar los temas fundamentales que son legítimamente integrados en el discurso de los participantes. Estas fuentes de información posibilitaron el análisis sistemático de lo que siente y piensa la gente del movimiento varias décadas después de haber participado en el mismo con respecto a (a) la incidencia de la educación en la adolescencia a largo plazo en sus propias vidas; (b) el movimiento juvenil que condujo a la autorrealización, entendida entonces por integrarse a un kibutz en Israel; (c) el Kibutz de hoy.

Estos temas fueron medidos a través de componentes que se refieren tanto al aspecto cognitivo del individuo al venir a tomar posición sobre temas específicos como a los aspectos expresivos del fenómeno (Adi, 2009).

\section{Nostalgia, realización y cotidianeidad}

La adolescencia es sin duda una época que queda grabada en la vida personal del individuo dado el impacto que tiene en la formación personal. En la historia de vida del individuo este periodo es indudablemente muy significativo tanto en relación a sus aspectos reales como imaginarios; es un periodo al que se le atribuyen vivencias únicas a las que muchas veces se añora y se quisiera repetir. Es el periodo en que en la búsqueda de la identidad personal las relaciones sociales con grupos de la misma edad tienen un impacto predominante (Erikson, 1968). El caso acá analizado ejemplifica la incidencia que tuvo el movimiento en la adolescencia de quienes permanecieron en él y decidieron seguir el rumbo indicado por la

10 Preferimos denominar a este aspecto del estudio, Narrative Analysis (y no Análisis Cualitativo, como hace cierta literatura). Lieblich, A. and others, Narrative Research Reading (1998). 
ideología predominante. Los hallazgos de la investigación muestran que la mayoría de los sujetos que respondieron al cuestionario evalúan el impacto esencial del movimiento en referencia al aporte a la formación personal. Ellos expresan lo que Boym (2001) considera una "nostalgia restaurativa" que implica un deseo de retornar al pasado para experimentar nuevamente las vivencias enriquecedoras de la época. Así el $90 \%$ de esta población no solo expresan recuerdos agradables de la época juvenil, sino que evalúan profundamente el aporte del movimiento en la formación de los valores humanos, en el despertar de la curiosidad intelectual y en el desarrollo personal en general. Por ejemplo, en las palabras de uno de los entrevistados "la participación en el movimiento juvenil dio significado a mi vida; nada, después de eso, tuvo el mismo significado". Otra entrevistada sostuvo que lo que lleva consigo de aquello días es ".... el magnetismo del movimiento juvenil, su honda influencia espiritual en la adolescencia que se prolonga durante toda la vida, su admirable don de tocar las mejores fibras morales en cada uno de nosotros..." y a quienes se refirieron al pasado afirmando que "desde los días del movimiento tengo satisfacciones en mi vida...solo por eso volvería a vivir todo de nuevo" o "...No solo volvemos para atrás, ese pasado está dentro de nosotros de por vida”.

Hay algunos entrevistados, aunque los menos, que desarrollaron hacia aquella época una "nostalgia de reflexión" (Boym, 2001), es decir una mirada más crítica hacia la experiencia vivida opinando, por ejemplo, que "...había una inocencia política tanto en nosotros como en los dirigentes..."; " los dirigentes creían en lo que decían, el problema es que ellos volaban en otras nubes". Pero también la mirada algo crítica no deja de enfatizar el aspecto emocional de la vivencia como, por ejemplo, en la frase de una entrevistada "... a pesar que el movimiento era dogmático y anacrónico no puedo dejar de valorar lo que me brindó... ", "el movimiento es más grande que todos sus errores..

¿Que recuerdan en realidad los ex miembros del movimiento? Un análisis más profundo de los resultados del cuestionario que permite ver las correlaciones entre las diferentes dimensiones presentadas muestran que los ex miembros del movimiento no se relacionan a la esencia ideológica de la institución (Adi, 2008). Ellos recuerdan vivencias sin confrontar a los sueños de 
entonces con la realidad cambiante que ya en aquellas décadas iba marcando un camino diferente del que los ideales propuestos prometían. Como dijo una de las pocas entrevistadas que atinaron a expresar una reflexión más crítica refiriéndose al camino equívoco marcado por el movimiento a través de los líderes "...que venían de Israel y seguían hablando del pionero socialista, el hombre de la tierra y no se relacionaban a los rápidos cambios que se iban produciendo en el medio a través del conocimiento y la tecnología” 11

En los hallazgos, las preguntas que hacían referencia a los contenidos transmitidos por el movimiento recibieron valores marginales en relación a la centralidad que recibió el movimiento como marco vivencial. Algunas de estas preguntas se centraban, por ejemplo, en las implicaciones de los objetivos ideológicos en cuanto a lo que se acostumbra a referir como "realización" o praxis ideológica. Los objetivos declarados por el movimiento no eran solo crear una institución para el desarrollo de una cultura juvenil más expresiva en base a valores sociales, sino que servía de marco para llevar adelante objetivos especificados en términos de prácticas muy concretas: emigrar a Israel e integrarse al kibutz. A consecuencia de la presión grupal, quienes no aceptaban los pasos así estipulados como camino de vida debían abandonar las filas del movimiento, independientemente de la profundidad de la vivencia. Aún más, quienes mantenían posiciones discrepantes en cuanto a la interpretación y la manera de lograr esos objetivos eran considerados disidentes e impulsados a abandonar la organización. De ahí lo sorprendente de los resultados del cuestionario: no existe correlación empírica entre la importancia del movimiento y los altos valores otorgados a la realización misma de sus objetivos. ¿Será que los participantes "se olvidaron” de lo fundamental y se aferraron solo a la nostalgia restaurativa a través de las “inolvidables vivencias?”.

Esta tendencia se puede reivindicar a través de las palabras de un ex miembro que no emigró a Israel y que resumió el impacto que tuvo sobre él un encuentro que se realizó para conmemorar los 60 años de la fundación del movimiento en Uruguay. Entre otras cosas el sostuvo que:

11 En el libro autobiográfico "Una Joven Política" la autora, que fue enviada por el movimiento a un curso de capacitación por un año a Israel 1 en la década del 50, ya se refiere al abismo entre los mensajes transmitidos en el movimiento y la realidad. 
"Muchos sueños quedaron por el camino. Los sesudos planteos ideológicos de otras épocas parecen extraños y trasnochados... Pero nos quedaron otras cosas del Hashomer de los años de la adolescencia: el amor a la naturaleza, el recuerdo de los primeros y tímidos amoríos, el descubrimiento del mundo de la poesía, la música y el arte, la fascinación de los grandes debates sobre hombres y libros, la emoción de las noches junto al fuego, el descubrimiento de la solidaridad colectiva, el planteo del constante desafío individual como forma de vida."

Esta nostalgia restaurativa pareciera aferrarse a la dimensión utópica de un esquema ideológico que, realizado o no, aún permite memorizar las vivencias independientemente de los objetivos no realizados. Es quizás esta posibilidad de reconstruir en la memoria los sueños del pasado lo que ayuda a dejar de lado el pensamiento crítico y a realzar la ligazón emocional hacia el ayer adolescente (Byan, 2001).

¿Entre los hallazgos de la investigación cabe destacar en este contexto la respuesta de los cuestionados a la pregunta "Con tu experiencia actual volverías a ingresar al movimiento juvenil?" Las respuestas manifiestan un alto consenso con respecto al rol que tuvo aquella experiencia en la vida de los participantes en la investigación: 91\% de los que aún permanecen en el kibutz y $88 \%$ de los que viven fuera del kibutz respondieron positivamente a la pregunta, lo que implica que estarían dispuestos a repetir la experiencia que les modeló la vida. ${ }^{12}$ Esta manera de recordar el movimiento parece más bien referirse a los sueños que a la realización de la ideología ya que las respuestas a la pregunta si volverían a vivir la experiencia del kibutz manifiesta un mayor disenso entre quienes respondieron al cuestionario.

\section{El kibutz de ayer, el kibutz de hoy}

El movimiento Hashomer Hatzair, a diferencia de otras organizaciones sionistas, no concebía la

12 En la introducción a su libro "La memoria del sueño" escribe Rubin las historias presentadas "Son el reflejo y la memoria de tiempos idos, en los que los sueños se mezclan con los ideales y la ideología para conformar esa rica vivencia que fue la tnuah (movimiento)". 
idea de emigrar a Israel sin vivir en el kibutz. Algunos de los entrevistados mencionaron que en el momento de la decisión lo que más los impulsaba no era el sionismo sino la posibilidad de vivir una vida comunitaria de igualdad total. "Si el kibutz no hubiera sido una opción real me hubiera quedado a vivir en Argentina donde la vida de ciudad era mucho más atractiva" comentó una interlocutora que emigró a Israel a mediado de los años 60 y abandonó el kibutz después de dos años de incorporarse a él.

Así, la vida de aquellos que emigraron hace cuatro, cinco o seis décadas, orientada por una ideología que cambió radicalmente, atravesó vuelcos configurados por el choque entre las expectativas y la realidad. Este proceso llevó a los pioneros de entonces a evaluar constantemente las decisiones que debían tomar en diferentes etapas de sus vidas para acomodarse a la tensión entre el kibutz que se fue transformando y acentuando los logros materiales y los sueños de una vida orientada a realzar los valores humanos de igualdad material, solidaridad, ayuda mutua, y demás (Bar Guil, 2007). El pensamiento utópico que alimentó la motivación de la inmigración dejó de jugar un papel dominante en la confrontación con la vida cotidiana. Su lugar fue ocupado por decisiones pragmáticas, capaces de responder a la necesidad de conformar una existencia cómoda y llevadera. La importancia atribuida a la integración a una forma de vida dictaminada por una realidad cambiante puede explicar parcialmente la gran decepción percibida por quienes creyeron realizar las prácticas derivadas de una visión ideal. El proceso de privatización, el salario diferencial, la apropiación individual de bienes, la administración del colectivo por medio de agentes externos son solo parte de los cambios que caracterizan al kibutz en las últimas décadas.

El desencanto así vivido se pone de manifiesto en las respuestas que dieron los ex miembros del movimiento a la pregunta: "con mi experiencia actual si podría volvería"... La pregunta se refería a los tres componentes centrales del cuestionario: Ingresar al movimiento juvenil, Radicarse en Israel e Ingresar al kibutz. Con respecto a los dos primeros temas hay un amplio consenso ya que $94 \%$ de los que aún permanecen en el kibutz respondieron positivamente y así lo hicieron también el 88\% de los que viven fuera 
del kibutz. Como fue explicado, la positiva actitud hacia participación en el movimiento es reafirmado a través de un imaginado "volver a vivir" reflejo de la nostalgia a los momentos en que la vivencia juvenil estaba encapsulada en normas de vida y valores que los distinguían profundamente del medio social en que vivían. En las palabras de una entrevistada que emigró a Israel en 1958 y aún vive en el kibutz "En el movimiento encontré compañeros y amigos nuevos, ideas nuevas, todo era diferente, eso me conquistó". La actitud con respecto al movimiento no se repite en lo que se relaciona al kibutz. Una gran proporción de los encuestados, que llegaron con la esperanza de modelar una sociedad diferente, evalúan con ambigüedad la praxis de esta ideología

El cuestionario que se entregó intentó evaluar la actitud de los interrogados con respecto al kibutz en varias dimensiones, de acuerdo al logro de los objetivos estipulados en la plataforma ideológica del movimiento. A cada uno de los objetivos se acordó una escala entre 1 y 10 donde el 1 se refiere al ideal que no se realizó y el 10 el ideal que se realizó. En lo referente a los objetivos ideológicos y sociales tales como la lucha por el socialismo o ayuda a los estratos sociales débiles en Israel el promedio otorgado al kibutz fue aproximadamente de 5 puntos. Una evaluación más positiva, con un promedio de 7 puntos, se acordó al rol del kibutz en el logro de objetivos nacionales. Estos se refieren tanto a cuestiones económicas (como tecnología y agricultura) como a temas relacionados a la absorción de inmigrantes, al sionismo, a la seguridad y otros aspectos del proceso de consolidación del estado.

En lo que se refiere al nivel personal, de tener que elegir nuevamente el camino que se anduvo en pro de los dos objetivos fundamentales que el movimiento juvenil consideraba como auto realización son evaluados por los ex miembros de manera divergente. En cuanto a la inmigración a Israel y a la concomitante adopción de la identidad israelí, 94\% de los encuestados respondieron que nuevamente elegirían ese camino “...ante mis ojos, es inmensamente valedero el poseer una identidad nacional que no se pone en duda,” afirmó un entrevistado. No así en lo que se refiere a vivir en el marco colectivo del kibutz. El 
arrepentimiento de este trayecto es manifestado por el $85 \%$ de los ex miembros del kibutz y por uno de cada cuatro de los encuestados aún miembros del kibutz que expresaron que con la experiencia actual no volverían a elegir ese destino. Un entrevistado, que abandonó el kibutz después de cinco años (1965-70) se expresó diciendo que "por un lado la idea del kibutz era una fuente de fuerza casi mágica para cambiar el orden social. aunque suena como exageración era hermoso creer ingenuamente”. En un acto en el que se conmemoró el 80 aniversario de la fundación del movimiento un participante en el debate sostuvo que él consideraba que la reunión se hacía para marcar tres de los grandes fracasos del movimiento ideológico. Refiriéndose al kibutz dijo: "reconozco mi tristeza y amargura al comprender que el kibutz va perdiendo su carácter colectivista. Ni siquiera puedo reconocer mi propio kibutz; trayendo las palabras del poeta "cuando duermo, en mis sueños yo sonrío; cuando despierto las lágrimas se deslizan por mi rostro"

El proceso de privatización que se fue dando en los kibutzim, a consecuencia de una gran crisis económica, contradice uno de los fundamentos básicos de igualdad que en los años juveniles atrajo a la población investigada. Ciertamente, el proceso no es idéntico en todos los kibutzim y hay aquellos que que privatizaron tanto la producción como el consumo mientras otros privatizaron todos los servicios y solo parte del consumo mientras otros, por lo general en estado económico mejor, privatizaron solo aspectos marginales. También en cuanto a la participación en el mercado de trabajo no existe un solo modelo que caracteriza a todos los kibutzim ya que los sueldos diferenciales dependen de la posibilidad de las capacidades de oferta al mercado. Además, muchos de los entrevistados ya estaban formalmente jubilados, lo que implicaba arreglos individuales de acuerdo a la situación del kibutz.

En el intento de entender la manera en que los entrevistados confrontaban la situación, se indagó con respecto a la manera en que ellos explican el proceso que se fue desarrollando. Hay quienes consideran a la privatización como la única opción de subsistir. Un miembro de un kibutz privatizado expresó que "Para salir de la pobreza comunista, tuvimos que adoptar los métodos capitalistas " ya que según otro entrevistado "el dilema era privatizar o dejar de existir". Indudablemente no todos los entrevistados ven el proceso como un 
imperativo para adaptarse a la realidad cambiante. Hay quienes prefieren enfrentarse a los cambios sin renunciar a los ideales que los llevaron a tomar la decisión de vivir en una sociedad creada en base a un modelo diferente del conocido en el mundo. Así, un pionero que vivía en un kibutz fundado por inmigrantes latinoamericanos en la primera década de la creación del Estado se expresó diciendo que:

-..no solo que el kibutz no fracasó, sino que...hay suficientes evidencias que indican que el kibutz persiste en la brecha histórica como orden social a imitar...estoy en conflicto con el proceso actual pero creo en la posibilidad de recuperación y por lo tanto me comporto de acuerdo a mi forma de ver el mundo....

Otra entrevistada, miembro del mismo kibutz, sostuvo una posición totalmente diferente que representa solo una pequeñísima minoría de quienes respondieron al cuestionario. Ella expresó su percepción del proceso diciendo que:

...de haber sabido cómo se desarrollaría el kibutz, no hubiera realizado alió (inmigración a Israel). No dedique mi vida para lo que veo hoy a mí alrededor. Debo decir claramente que nos equivocamos. Esto fracasó... el kibutz no tiene futuro, a la larga se convertirá en una comunidad organizada como tantas otras.

Tal como fue explicada esta tendencia no caracteriza las opiniones de muchos de los participantes en la investigación. Dentro del 41 por ciento de quienes se encuentran en el kibutz con privatización parcial más de un $70 \%$ consideran que el kibutz podrá recuperar su forma de vida basada en las premisas ideológicas. Así por ejemplo una entrevistada que vive en el kibutz desde el año 1958 afirmó que la solución de la privatización permitió al kibutz recuperarse económicamente, “...aun así yo veo un futuro en la recuperación del modelo original del kibutz; siempre habrá gente que busca una sociedad mejor...". "Es un desafío tratar de crear modelos que sean pragmáticos sin dejar de lado los valores humanos", sostuvo otro entrevistado. 
Considerando las Ínter correlaciones obtenidas en el análisis de los cuestionarios a través del SSA se perfila que los temas relacionados al kibutz como ente ideológicamente igualitario casi no tienen relación con los recuerdos positivos y emotivos hacia el movimiento juvenil. La decepción con respecto a la ideología del kibutz clásico se manifiesta en diferentes términos tanto al nivel personal afectivo (desilusión profunda) como a nivel pragmático (inseguridad económica en la vejez) como institucional (errores muy grandes en las decisiones de políticas de desarrollo).

En general, el aspecto expresivo más que el cognitivo-ideológico, es el que parece explicar el por qué un $50 \%$ de los miembros del kibutz que respondieron al cuestionario marcaron positivamente la respuesta que se quedan en el kibutz porque así lo desean. Esta respuesta tiene alta correlación con la agradabilidad de vivir en ese ambiente. No hay ninguna correlación estadística con la idea de igualdad o con el hecho que el kibutz atraviesa por el proceso de privatización. Lo que los entrevistados ponen claramente de manifiesto es que el concepto de solidaridad no implica identificación con las capas sociales débiles de la sociedad, tal como se sostenía en la ideología del movimiento. El concepto es traducido en términos de ayuda al prójimo dentro del marco de la comunidad del kibutz, es decir el saber que hay quien pueda apoyar en momentos de dificultades personales tales como enfermedad, duelo, u otros problemas. Esta tendencia demuestra que el marco de referencia se fue transformando y se fue reduciendo a la coexistencia cotidiana más que a los objetivos macro sociales planteados por el movimiento. Aun así, hay quien sostuvo que “...en "grande " el kibutz realizó las aspiraciones del movimiento, aunque se haya alejado del modelo clásico..." Hacia esta orientación apuntan 59\% de los entrevistados que abandonaron el kibutz en distintas etapas de sus vidas y sostienen que los ideales fundamentales del movimiento se realizaron.

Independiente de la evaluación de los logros del kibutz, parte de las preguntas estaban orientadas a evaluar la satisfacción de la vida en general de los ex miembros del movimiento. Los hallazgos muestran que casi no hay diferencia entre ambas poblaciones ya que un $90 \%$ se siente bastante o muy satisfecho de la vida en general. Es decir, el hecho que el kibutz haya sido o no consecuente con el modelo al cual se aspiraba, no afectó el 
bienestar subjetivo de los actores. Una pequeña diferencia en los resultados se percibe en cuestiones relacionadas a aspectos particulares de la vida que se relacionan puntualmente a situaciones relacionadas con el bienestar económico tales como seguridad en la vejez, la posibilidad de ayudar a los hijos y la evaluación de los ingresos. En este tema los miembros del kibutz se sienten un poco menos satisfechos que quienes abandonaron. Pero aun así a través de las entrevistas no se percibe un dejo de amargura o descontento con la situación de vida.

\section{Revivir el pasado, reordenar el presente}

Los ex miembros del movimiento juvenil indudablemente tomaron un compromiso con la ideología sionista socialista y convirtieron las ideas en prácticas de vida: emigraron a Israel y vivieron o viven en el kibutz. Ambos objetivos estaban unidos en la narrativa juvenil que orientó a aquellos jóvenes a tomar decisiones tan radicales en la vida personal (Siebzehner, 2013). Enfrentados con la evaluación del camino que emprendieron décadas después, los actores restauran las vivencias y experiencias que los llevaron a esas decisiones más que la ideología que los orientó. A través de la nostalgia ellos reafirman la importancia del camino que eligieron o como lo testimonian algunos entrevistados "aquellos ideales no realizados no borran la satisfacción de haber vivido por ellos..." “... el movimiento juvenil fue deslumbrante para mí.... incluso si el hechizo fue una ilusión, me residía agradable revivirlo..." En este proceso del despertar recuerdos los entrevistados reordenan la importancia de los mensajes centrales a los que se adhirieron en la juventud creando divergencia entre ambos. La "ingenuidad", la "inmadurez", "la equivocación”, "la utopía" son atribuidas a la forma de vida colectiva que intentaron realizar y que según algunos concluyó su rol histórico. La dimensión nacional, sin embargo, juega para más del 90\% de los cuestionados un rol fundamental y trascendente. El adoptar la identidad israelí, el educar a los hijos en un medio judío, el haber aportado a la construcción del Estado, el sentirse integrado en la sociedad, parecen superar y disminuir la importancia del impacto marginal del kibutz a largo plazo tanto en la sociedad como en sus vidas personales. Los logros, entonces, pueden ser atribuidos al movimiento mismo. En las palabras de un entrevistado que llegó al país en 1951 y vive aún en el kibutz 
"Mira lo que el movimiento construyó en la Galilea, en el Negev y en el centro del país; mira el extraordinario comportamiento de sus miembros en todos los frentes de batalla a lo largo de los años. Esto es el ejemplo más venerable de la lealtad al pueblo judío y al Estado de Israel...

Entonces ¿por qué no querer integrarse nuevamente si se volviese en el tiempo? La nostalgia reconstructiva es quizás lo que permitió a uno de los ex activistas caracterizar al movimiento juvenil como una "Inmaculada decepción". El lazo emocional con un pasado irreversible parece rechazar la posibilidad de crítica o es quizás el tiempo y el esfuerzo invertidos en la posibilidad de ver surgir un mundo mejor permiten dejar enredar lo imaginario con lo real; lo logrado con lo que se quería lograr; los avatares de una realidad cambiante.

\section{Bibliografía}

Adi, P. P. y B. Siebzehner, (2008). Los idealistas y el kibutz ante la realidad. Kfar Saba: Beit Berl (en hebreo).

Adi, P. (2009) “Avatares de un sueño. La inmigración de los pioneros latinoamericanos a Israel”, El Olivo, 69-70: 129-154.

Adler, Ch y Y. Perez (1968) "Los movimientos juveniles y la sociedad de salones", en Sh. N. Eisenstadt y otros, Educación y Sociedad en Israel. Jerusalén: Akademon. (en hebreo).

Alón, M., (1986). La posibilidad eterna: Juventud y cambio social. Tel Aviv: Sifriat Hapoalim (en hebreo)

Ben David, Y. (1954). "Membresía en los movimientos juveniles y el status social”,Megamot, H,3: 227-247,

Ben Rafael, E., (1996). La Revolución Incompleta. Tel Aviv: Yad Tabenkin (en hebreo),

Bar Guil Sh. (2005). Al principio había un sueño: los veteranos de los movimientos juveniles pioneros de América Latina. Be'er Sheva: Universidad Ben Gurion en el Negev (en hebreo),

Bar Guil Sh. (2007). Adolescencia-Visión y Realidad. Del Dror y Gordonia hasta el Ijud Habonim en Argentina 1934-1973. Tel Aviv: Yad Tabenkin (en hebreo),

Boym, S. (2001). The future of nostalgia. New York, NY: Basic Books,

Chen, M (1969). Educational Concomitants of Adolescent Participation in Israelí Youth Organization, Research Report. Jerusalem: The Szold Institute, 
Erikson, Erik H. (1968) Identity, Youth and Crisis. New York: Norton.

Giddens, A. (1999). Modernity and Self-Identity: Self and Society in the Late Modern Age. Cambridge: Polity Press,

Golan, O., (1998). La expresión juvenil en la época postmoderna: el caso de "la admiración". Jerusalén: Centro Shain, Universidad Hebrea de Jerusalén (en hebreo),

Guttman, L. (1980) Role of Facets in Regional and Dimensionality Hypothesis os Smallest Space Analysis. Jerusalem: Institute of Applied Social Research,

Kahane, R., (2007). Juventud y el código informal. Los movimientos juveniles en el siglo XX y las fuentes de la juventud postmoderna. Jerusalén: Mosad Bialik (en hebreo),

Klor, S. (2016). "Marginal Immigrants: Jewish Argentina Immigration to the State of Israel, 19481967” Israel Studies, 21, 2: 50-76,

Lam, T., 1991). Movimientos Juveniles Sionistas en una mirada retrospectiva. Haifa: Sifriat Poalim (en Hebreo),

Levy, S. (ed.) (1994). Louis Guttman on Theory and Methodology: Selected Writings. Aldershot, UK: Dartmouth, 
Lieblich, A., Tuval-Mashiach, R. and Zilber, T, (1998) Narrative Research Reading, Analysis and Interpretation. Thousand Oaks CA: Sage,

Rapoport, T., Lomsky Feder y otros (1995) “Jóvenes y juventud en la sociedad israelí", en Flus, Ch. (editor) Adolescentes en Israel: Aspectos personales, familiares y sociales. Even Yehuda: Reches (en hebreo),

Rubín, Y., (2006). La Memoria del Sueño. Buenos Aires: Colección Imaginaria, A.M.I.A,

Schechner, D. (2002) Una muchacha política. Tel Aviv: Ha Kibutz Hameuchad,

Siebzehner, B. (2013) "Cautivos por la ideología: miembros de los movimientos juveniles miran al Israel de entonces y de hoy" Judaica Latinoamericana, VII: 511-535,

Stoliar, I. (2004). Los Guardianes (shomrim) del país de los Andes. Givat Haviva: Yad Yaari,

Stoliar I. (2013). Los Guardianes (shomrim) del Caribe. Historia de Hashomer Hatzair en Cuba. Givat Haviva: Yad Yaari,

Verner, A. (1996). Guardian (Shomer) una vez. Tel Aviv: Ediciones Or (en hebreo). 